\title{
Spinal Injury Associated With Firearm Use
}

\author{
Randall T. Loder ${ }^{1}$, Abhipri Mishra ${ }^{2}$, Bradley Atoa ${ }^{2}$, Allison Young ${ }^{2}$ \\ 1. Orthopaedic Surgery, Riley Hospital for Children, Indianapolis, USA 2. Orthopaedic Surgery, Indiana University \\ School of Medicine, Indianapolis, USA
}

Corresponding author: Randall T. Loder, rloder@iupui.edu

\section{Abstract \\ Objective}

Injuries associated with firearms are a significant health burden. However, there is no comprehensive study of firearm spinal injuries over a large population. It was the purpose of this study to analyze the demographics of spinal firearm injuries across the entire United States for all ages using a national database.

\section{Methods}

A retrospective review of prospectively collected data using the Inter-University Consortium for Political and Social Research Firearm Injury Surveillance Study 1993-2015 (ICPSR 37276) was performed. The demographic variables of patients with spinal injuries due to firearms were analyzed with statistical analyses accounting for the weighted, stratified nature of the data, using SUDAAN $11.0 .01^{\mathrm{TM}}$ software (RTI International, Research Triangle Park, North Carolina, 2013). A p-value of $<0.05$ was considered statistically significant.

\section{Results}

For the years 1993 through 2015, there were an estimated 2,667,896 emergency department (ED) visits for injuries due to firearms; 10,296 of these injuries $(0.4 \%)$ involved the spine. The vast majority $(98.2 \%)$ were due to powder firearm gunshot wounds. Those with a spine injury were more likely to have been injured in an assault ( $83.7 \%$ vs. $60.2 \%$ ), involved a handgun ( $83.5 \%$ vs. $60.2 \%)$, were male ( $90.8 \%$ vs. $86.4 \%$ ), were admitted to the hospital ( $86.8 \%$ vs. $30.9 \%$ ), and were seen in urban hospitals ( 86.7 vs. $64.6 \%$ ). The average age was 28 years with very few on those $<14$ years of age. Illicit drug involvement was over four times as frequent in those with a spine injury (34.7\% vs. $8.0 \%$ ). The cervical spine was involved in $30 \%$, thoracic in $32 \%$, lumbar in $32 \%$, and sacrum in $6 \%$. A fracture occurred in $91.8 \%$ and neurologic injury in $33 \%$. Injuries to the thoracic spine had the highest percentage of neurologic involvement (50.4\%). There was an annual percentage decrease for patients with and without spine involvement in the 1990s, followed by increases through 2015. The average percentage increase for patients with a spine injury was 10.3\% per year from 1997 onwards $\left(\mathrm{p}<10^{-6}\right)$, significantly greater than the $1.5 \%$ for those without spinal involvement $(\mathrm{p}=0.0001)$ from 1999 onwards.

Review began 02/28/2021 Review ended 03/14/2021 Published 03/16/2021

\section{() Copyright 2021}

Loder et al. This is an open access article distributed under the terms of the Creative Commons Attribution License CC-BY 4.0., which permits unrestricted use, distribution, and reproduction in any medium, provided the original author and source are credited.

\section{Conclusions}

This nation-wide study of spinal injuries associated with firearms covering all ages can be used as baseline data for future firearm studies. A reduction in the incidence of such injuries can be guided by our findings but may be difficult due to sociopolitical barriers (e.g. socioeconomic status of the injured patients, differences in political opinion regarding gun control in the US, and geospatial patterns of firearm injury).

Categories: Epidemiology/Public Health, Trauma

Keywords: firearm, injury, spine, neurologic injury, cervical, thoracic, lumbar, demographics

\section{Introduction}

Injuries associated with firearms are a significant health burden [1-3]. While firearm injuries represent only $4 \%$ of injuries seen at major trauma centers (National Trauma Databank information), deaths attributed to firearms in the population are equivalent to those from motor vehicle crashes and falls [2]. They also result in significant costs to society, both financially and in loss of human life/work [3-5]. Firearm injuries account for more than an annual $\$ 70$ billion in costs [3] to the US health care system. Ranney [4] noted that in the six months after a firearm injury, patient-level health care visits and costs increased three to 20 times when compared to the six months prior. They also account for the sizeable human loss of life [5]; for those with a gunshot spinal cord injury due, the life expectancy loss for each person with quadriplegia is 17 years and with paraplegia 11.4 years. This equates to 25,647 years of life lost each year due to new spinal cord gunshot injuries.

There is some literature regarding firearm injuries to the spine, however many focus on only one anatomic area (e.g., cervical), multiple mechanisms of injury, including firearms, specific age groups, war injuries, 
general reviews regarding treatment, or case reports. Furthermore, there are no comprehensive studies of firearm spinal injuries over a large population. The aim of this study was to analyze injuries to the spine due to firearm activity across the entire United States for all ages using a national database. Such data will be useful as baseline data for future studies regarding spinal injuries due to firearms and can serve as a guide for injury prevention programs. This also begins to fill a void in the paucity of firearm research, which has been recently noted [6].

\section{Materials And Methods}

The data for this study were obtained from the Inter-University Consortium for Political and Social Research Firearm Injury Surveillance Study 1993-2015 (ICPSR 37276) [7] collected by the National Electronic Injury Surveillance System (NEISS). The NEISS, a branch of the US Consumer Product Safety Commission, collects data from a probability sample of hospitals in the United States and its territories that have at least six beds and an emergency department (ED). The sample contains five strata: four based on size (the total number of emergency room visits reported by the hospital and are small, medium, large, and very large) and one consisting of children's hospitals. The NEISS is composed of $\sim 100$ hospitals, as this number varies slightly from year to year. Patient information is collected daily from each NEISS hospital for every patient treated in the ED due to an injury associated with consumer products. For this particular study, the ICPSR data set consists of any patient seeking care in the ED for any firearm-related injury, regardless of activity involved during the injury (e.g. hunting, committing a crime, suicide, assault), and whether or not the patient sustained a gunshot wound (coded as GSW by NEISS) or injured in some other way (coded as NGSW by NEISS). Examples of an NGSW are a laceration while cleaning a firearm, head trauma from being pistolwhipped, a clavicle fracture from a rifle recoil, etc. Further details regarding the acquisition of ICPSR/NEISS data and guidelines for use of such data can be accessed from their respective websites (ICPSR www.icpsr.umich.edu, NEISS -www.cpsc.gov/library/neiss.html).

The data for 1993 through 2015 due to firearms were downloaded from the ICPSR website. This data set includes age/age groups, injury diagnosis, gender, race, marital status, type of firearm, the perpetrator of injury, intent of injury (unintentional, assault, suicide, law enforcement), anatomic location of the injury, method of transportation to the ED, disposition from the ED, the involvement of drugs/crimes/fights/arguments in the incident, and whether or not the patient was shot. The race was classified as White, Black, Amerindian (Hispanic and Native American), and Indo-Malay (Asian origin) [8]. This study was considered exempt by our local institutional review board.

Injuries involving the spine were ascertained by reviewing the cases and narrative comments for those with a BDYPT (body part) code of 31 (upper trunk), 79 (lower trunk), and 89 (neck) and using the diagnosis codes of fracture (57), internal organ injury (62), and nerve damage (61). Next, all the narrative comments were searched using the FIND command in Microsoft Excel ${ }^{\mathrm{TM}}$ (Microsoft ${ }^{\circledR}$ Office 365, Microsoft Corporation, Redmond, WA)) using the keywords: vert, sacr, cocc, thor, lumbar, cerv, atlas, axis, quad, para, as well as each individual vertebra (ie. C1, 2, . , L5). A neurologic injury was considered present when the diagnosis code was 61 (nerve damage) and/or when the search of the narrative comments was positive for paraplegia, quadriplegia, or paralyzed/paralysis and when the diagnosis code 62 (internal organ injury) was associated with a neurologic injury in the narrative comments. The NEISS does not report an American Spinal Injury Association Impairment Scale or Injury Severity Score.

We also wished to analyze the prevalence of sexual assault and alcohol involvement with these events. Sexual assault was determined by searching for the keywords of rape, sex, sexual assault, incest, sodomy, intercourse, ejaculate, penetration, vagin, oral, and anal. Alcohol involvement was determined by searching for the keywords alcohol, EtOH, intoxicated, drinking, drank, drunk, club, ethanol, saloon, tavern, liquor, booze, beer, whiskey, brandy, rum, vodka, scotch, tequila, wine, sake, champagne, cognac, and BAC (an acronym for blood alcohol involvement).

\section{Statistical analysis}

Statistical analyses were performed with SUDAAN $11.0 .01^{\mathrm{TM}}$ software (RTI International, Research Triangle Park, North Carolina, 2013) to account for the stratified and weighted nature of the data. The estimated number of ED visits was calculated, along with $95 \%$ confidence intervals (CIs) of the estimate. (Throughout the remainder of the manuscript when numbers are denoted as $\{x, y\}$, these represent the $95 \%$ CIs of the estimate). When the actual number of patients $(n)$ is $<20$, the estimated number $(N)$ becomes unstable and should be interpreted with caution; thus both $\mathrm{n}$ and $\mathrm{N}$ were reported. Analyses between groups of continuous data were performed with the t-test (two groups) or analysis of variance (ANOVA) (three or more groups). Differences between groups of categorical data were analyzed by the $\mathrm{c} 2$ test. Joinpoint regression analysis was used to analyze for percentage changes over time (Joinpoint Regression Program, Version 4.8.0.1, April 2020; Statistical Research and Applications Branch, National Cancer Institute [https://surveillance.cancer.gov/joinpoint/]). For all analyses, a $\mathrm{p}<0.05$ was considered statistically significant.

\section{Results}




\section{Analyses between patients and without a spine injury}

Patients with a spine injury (Table 1 ) were more likely to have been injured during an assault ( $83.7 \%$ vs. $60.2 \% ; p=0.0009)$, involved a handgun ( $83.5 \%$ vs. $60.2 \% ; p=0.0001)$, male sex ( $90.8 \%$ vs. $86.4 \% ; p=0.003$ ), admitted to the hospital ( $86.8 \%$ vs. $30.9 \%$; $p=0.0006)$, and seen in larger hospitals ( 86.7 vs. $64.6 \% ; p=$ 0.006). The injury was less commonly self-inflicted $(9.9 \%$ vs. $25.9 \%$; $=0.028)$. Although there was no overall difference in the average age between the patients with a spinal injury compared to those without ( 28.1 vs. 27.8 years; $p=0.67$ ), there was a marked difference when broken down by age groups, with very few spinal injuries in patients $<14$ years of age (Figure 1). Illicit drug involvement was over four times as frequent in patients with a spine injury ( $34.7 \%$ vs. $8.0 \%$; $=0.0052)$, and involvement in a crime was 1.5 times as frequent $(40.6 \%$ vs. $27.6 \% ; \mathrm{p}=0.046)$ in patients with a spine injury. There were no sexual assaults in the spinal injury group.

\begin{tabular}{|c|c|c|c|c|c|c|c|c|c|c|c|}
\hline & \multicolumn{5}{|c|}{ Spine involvement } & \multicolumn{5}{|c|}{ No spine involvement } & \multirow[b]{2}{*}{$p$ value } \\
\hline & $\mathrm{n}$ & $\mathrm{N}$ & L95\% & U95\% & $\%$ & $\mathrm{n}$ & $\mathrm{N}$ & L95\% & U95\% & $\%$ & \\
\hline All & 420 & 10,296 & 7,205 & 14,944 & 0.4 & 90,720 & 2,658,361 & $2,653,713$ & $2,661,452$ & 99.6 & \\
\hline \multicolumn{12}{|l|}{ Age (years) } \\
\hline Mean $[95 \% \mathrm{Cl}]$ & \multicolumn{5}{|c|}{$28.1[26.6,29.5]$} & \multicolumn{4}{|c|}{$27.8[27.1,28.4]$} & & 0.67 \\
\hline Median [interquartile] & \multicolumn{5}{|c|}{$23.5[19.3,33.2]$} & \multicolumn{5}{|c|}{$23.5[17.7,34.1]$} & \\
\hline \multicolumn{12}{|l|}{ Injury intent } \\
\hline Unintentional & 22 & 776 & 426 & 1,372 & 7.9 & 19,998 & 790,532 & 638,015 & 959,658 & 33.0 & 0.0009 \\
\hline Assault & 349 & 8,192 & 7,433 & 8,742 & 83.7 & 56,317 & $1,441,298$ & $1,259,506$ & $1,612,043$ & 60.2 & \\
\hline Suicide & 18 & 580 & 318 & 1,035 & 5.9 & 3,774 & 131,815 & 95,559 & 180,579 & 5.5 & \\
\hline Law enforcement & 11 & 241 & 144 & 401 & 2.5 & 932 & 31,308 & 22,992 & 42,630 & 1.3 & \\
\hline \multicolumn{12}{|l|}{ Firearm type } \\
\hline Handgun & 104 & 2,617 & 2,278 & 2,839 & 83.5 & 24,781 & 701,369 & 587,132 & 816,930 & 48.3 & 0.0001 \\
\hline Rifle & 8 & 287 & 145 & 541 & 9.2 & 3,796 & 145,263 & 110,105 & 189,997 & 10.0 & \\
\hline Shotgun & 5 & 224 & 60 & 732 & 7.1 & 3,256 & 131,436 & 112,865 & 152,666 & 9.0 & \\
\hline BB & 1 & 6 & 1 & 47 & 0.2 & 12,680 & 474,511 & 391,615 & 565,634 & 32.7 & \\
\hline \multicolumn{12}{|l|}{ Hospital size } \\
\hline Small & 7 & 589 & 228 & 1,441 & 5.7 & 6,476 & 507,349 & 363,664 & 690,642 & 19.1 & 0.0061 \\
\hline Medium & 15 & 690 & 257 & 1,727 & 6.7 & 7,430 & 406,425 & 275,406 & 584,308 & 15.3 & \\
\hline Large & 69 & 3,988 & 1,511 & 7,198 & 38.7 & 13,608 & 758,313 & 404,071 & $1,251,025$ & 28.5 & \\
\hline Very large & 313 & 4,937 & 2,426 & 7,553 & 48.0 & 58,525 & 958,628 & 643,589 & $1,326,522$ & 36.1 & \\
\hline Children's & 16 & 92 & 38 & 222 & 0.9 & 4,681 & 27,646 & 18,077 & 42,534 & 1.0 & \\
\hline \multicolumn{12}{|l|}{ Sex } \\
\hline Male & 379 & 9,332 & 8,950 & 9,612 & 90.8 & 78,802 & $2,295,005$ & $2,267,481$ & 2,320,634 & 86.4 & 0.0033 \\
\hline Female & 39 & 944 & 664 & 1,326 & 9.2 & 11,888 & 362,615 & 336,986 & 390,139 & 13.6 & \\
\hline \multicolumn{12}{|l|}{ Race } \\
\hline White & 80 & 2,830 & 2,241 & 3,481 & 35.1 & 23,843 & 931,455 & 740,477 & $1,133,254$ & 42.6 & 0.059 \\
\hline Black & 146 & 3,053 & 1,803 & 4,540 & 37.9 & 38,409 & 872,340 & 626,255 & 1,144,195 & 39.9 & \\
\hline Amerindian & 64 & 2,072 & 1,193 & 3,287 & 25.7 & 9,368 & 363,148 & 203,062 & 610,719 & 16.6 & \\
\hline Asian & 6 & 98 & 35 & 271 & 1.2 & 866 & 21,228 & 11,378 & 39,606 & 1.0 & \\
\hline \multicolumn{12}{|l|}{ Incident locale } \\
\hline Home & 68 & 1,969 & 1,510 & 2,461 & 41.6 & 20,327 & 732,937 & 631,178 & 836,432 & 47.1 & 0.011 \\
\hline
\end{tabular}




\begin{tabular}{|c|c|c|c|c|c|c|c|c|c|c|c|}
\hline School/recreation & 14 & 354 & 153 & 775 & 7.5 & 1,986 & 78,502 & 64,005 & 96,242 & 5.0 & \\
\hline Street/highway & 53 & 1,265 & 848 & 1,792 & 26.7 & 18,310 & 434,890 & 316,601 & 576,828 & 27.9 & \\
\hline Other property & 47 & 1,144 & 834 & 1,523 & 24.2 & 10,250 & 304,040 & 252,129 & 363,632 & 19.5 & \\
\hline Farm & 0 & 0 & 0 & 0 & 0.0 & 127 & 6,942 & 4,360 & 11,057 & 0.4 & \\
\hline \multicolumn{12}{|l|}{ Transportation to ED } \\
\hline Emergency medical service & 349 & 8,414 & 7,860 & 8,843 & 84.4 & 49,921 & $1,255,959$ & $1,049,318$ & $1,458,588$ & 52.8 & 0.0001 \\
\hline Air & 27 & 808 & 473 & 1,346 & 8.1 & 1,917 & 58,535 & 34,462 & 98,396 & 2.5 & \\
\hline Private vehicle & 13 & 521 & 272 & 976 & 5.2 & 17,930 & 698,677 & 545,218 & 874,630 & 29.4 & \\
\hline Walk-in & 5 & 144 & 64 & 321 & 1.4 & 8,161 & 283,043 & 203,446 & 388,355 & 11.9 & \\
\hline Police & 5 & 79 & 15 & 418 & 0.8 & 2,977 & 72,219 & 35,413 & 145,217 & 3.0 & \\
\hline Other & 0 & 0 & 0 & 0 & 0.0 & 237 & 8,278 & 4,040 & 16,399 & 0.3 & \\
\hline \multicolumn{12}{|l|}{ Anatomic location of injury } \\
\hline Head/neck & 126 & 3,015 & 3,593 & 306,716 & 29.6 & 25,521 & 793,383 & 740,891 & 847,663 & 30.5 & 0.0004 \\
\hline Upper trunk & 138 & 3,605 & 4,305 & 366,737 & 35.4 & 14,266 & 381,696 & 334,378 & 434,639 & 14.7 & \\
\hline Lower trunk & 148 & 3,427 & 3,894 & 348,629 & 33.7 & 11,123 & 289,804 & 257,815 & 325,003 & 11.1 & \\
\hline Upper extremity & 1 & 82 & 610 & 8,342 & 0.8 & 14,783 & 491,967 & 442,972 & 545,057 & 18.9 & \\
\hline Lower extremity & 4 & 44 & 137 & 4,476 & 0.4 & 23,075 & 647,338 & 615,109 & 680,735 & 24.9 & \\
\hline \multicolumn{12}{|l|}{ Diagnosis } \\
\hline Contusion/abrasion & 0 & 0 & 0 & 0 & 0.0 & 5,069 & 166,538 & 142,625 & 194,201 & 6.3 & $<10-4$ \\
\hline Foreign body & 23 & 576 & 1,222 & 59,305 & 5.6 & 9,135 & 323,836 & 248,409 & 417,875 & 12.3 & \\
\hline Fracture & 163 & 3,752 & 4,747 & 386,306 & 36.4 & 6,523 & 188,859 & 157,624 & 225,779 & 7.2 & \\
\hline Laceration & 34 & 839 & 1,410 & 86,383 & 8.1 & 9,807 & 335,217 & 263,935 & 422,348 & 12.7 & \\
\hline Internal organ injury & 61 & 1,093 & 2,413 & 112,535 & 10.6 & 4,640 & 127,629 & 99,469 & 163,413 & 4.9 & \\
\hline Puncture & 54 & 2,974 & 4,282 & 306,203 & 28.9 & 28,777 & 840,801 & 671,810 & $1,029,951$ & 32.0 & \\
\hline Not stated & 45 & 1,062 & 1,964 & 109,344 & 10.3 & 26,026 & 648,574 & 497,345 & 827,855 & 24.6 & \\
\hline \multicolumn{12}{|l|}{ ED Disposition } \\
\hline Treated and released & 35 & 1,309 & 793 & 2,086 & 12.8 & 51,326 & $1,686,619$ & $1,503,591$ & $1,855,563$ & 64.0 & 0.0006 \\
\hline Admit & 382 & 8,873 & 8,097 & 9,394 & 86.8 & 33,742 & 813,584 & 664,132 & 979,984 & 30.9 & \\
\hline Fatal & 2 & 37 & 9 & 145 & 0.4 & 4,910 & 136,289 & 115,742 & 160,299 & 5.2 & \\
\hline \multicolumn{12}{|l|}{ Marital Status } \\
\hline Never married & 150 & 4,017 & 3,488 & 4,442 & 74.0 & 33,207 & 927,325 & 810,059 & $1,029,794$ & 68.0 & 0.20 \\
\hline Married & 26 & 910 & 585 & 1,364 & 16.8 & 7,619 & 308,953 & 244,696 & 384,366 & 22.7 & \\
\hline Divorced/separated & 7 & 173 & 96 & 308 & 3.2 & 1,564 & 60,117 & 44,329 & 81,156 & 4.4 & \\
\hline Other & 6 & 327 & 87 & 1,093 & 6.0 & 1,239 & 67,661 & 24,961 & 173,906 & 5.0 & \\
\hline \multicolumn{12}{|l|}{ Argument } \\
\hline Yes & 30 & 515 & 193 & 1,126 & 19.6 & 5,440 & 165,558 & 136,973 & 198,871 & 15.0 & 0.54 \\
\hline No & 69 & 2,115 & 1,504 & 2,437 & 80.4 & 25,064 & 941,741 & 908,428 & 970,326 & 85.0 & \\
\hline \multicolumn{12}{|l|}{ Crime } \\
\hline Yes & 61 & 1,319 & 908 & 1,775 & 40.6 & 15,238 & 352,085 & 230,102 & 507,398 & 27.6 & 0.046 \\
\hline
\end{tabular}




\section{Cureus}

\begin{tabular}{|c|c|c|c|c|c|c|c|c|c|c|c|}
\hline No & 56 & 1,926 & 1,470 & 2,337 & 59.4 & 13,695 & 923,426 & 768,113 & $1,045,409$ & 72.4 & \\
\hline \multicolumn{12}{|l|}{ Illicit drug involvement } \\
\hline Yes & 35 & 958 & 709 & 1,242 & 34.7 & 2,457 & 83,146 & 46,498 & 144,475 & 8.0 & 0.0052 \\
\hline No & 60 & 1,804 & 1,520 & 2,053 & 65.3 & 25,781 & 954,749 & 893,420 & 991,397 & 92.0 & \\
\hline \multicolumn{12}{|l|}{ Fight } \\
\hline Yes & 36 & 872 & 555 & 1,291 & 25.1 & 7,768 & 229,151 & 189,007 & 275,510 & 19.0 & 0.20 \\
\hline No & 83 & 2,609 & 2,190 & 2,926 & 74.9 & 25,879 & 973,951 & 927,592 & $1,014,095$ & 81.0 & \\
\hline \multicolumn{12}{|l|}{ Alcohol involvement } \\
\hline Yes & 29 & 818 & 521 & 1,261 & 7.9 & 4,081 & 143,694 & 87,538 & 236,836 & 5.4 & 0.056 \\
\hline No & 391 & 9,478 & 9,035 & 9,775 & 92.1 & 86,636 & $2,514,519$ & $2,448,377$ & $2,597,675$ & 93.6 & \\
\hline \multicolumn{12}{|l|}{ Sexual assault } \\
\hline Yes & 0 & 0 & 0 & 0 & 0.0 & 505 & 13,067 & $2,640,018$ & $2,649,057$ & 0.5 & 0.0012 \\
\hline No & 420 & 10,296 & 7,205 & 14,944 & 100.0 & 90,214 & $2,645,278$ & 0 & 0 & 99.5 & \\
\hline \multicolumn{12}{|l|}{ Who caused } \\
\hline Unknown & 260 & 5,773 & 4,879 & 6,578 & 56.3 & 44,887 & $1,103,518$ & 971,206 & $1,227,885$ & 41.7 & 0.028 \\
\hline Stranger & 54 & 1,400 & 1,083 & 1,776 & 13.7 & 13,311 & 369,553 & 314,307 & 428,240 & 14.0 & \\
\hline Self & 30 & 1,017 & 627 & 1,596 & 9.9 & 17,077 & 683,502 & 558,034 & 817,357 & 25.9 & \\
\hline Friend/acquaintance & 19 & 520 & 281 & 935 & 5.1 & 5,425 & 180,846 & 152,792 & 211,476 & 6.8 & \\
\hline Spouse/ex & 2 & 59 & 12 & 281 & 0.6 & 522 & 19,137 & 15,332 & 23,527 & 0.7 & \\
\hline Other relative & 5 & 135 & 60 & 294 & 1.3 & 2,065 & 76,853 & 61,593 & 94,636 & 2.9 & \\
\hline \multirow[t]{3}{*}{ Other/not seen } & 50 & 1,392 & 1,008 & 1,875 & 13.6 & 7,433 & 224,952 & 184,778 & 270,161 & 8.5 & \\
\hline & \multicolumn{5}{|c|}{ Spine involvement } & \multicolumn{5}{|c|}{ No spine involvement } & \\
\hline & $\mathrm{n}$ & $\mathrm{N}$ & L95\% & U95\% & $\%$ & $\mathrm{n}$ & $\mathrm{N}$ & L95\% & U95\% & $\%$ & $p$ value \\
\hline All & 420 & 10,296 & 7,205 & 14,944 & 0.4 & 90,720 & $2,658,361$ & $2,653,713$ & $2,661,452$ & 99.6 & \\
\hline Age (years) & & & & & & & & & & & \\
\hline Mean $[95 \% \mathrm{Cl}]$ & \multicolumn{5}{|c|}{$28.1[26.6,29.5]$} & \multicolumn{4}{|c|}{$27.8[27.1,28.4]$} & & 0.67 \\
\hline Median [interquartile] & \multicolumn{5}{|c|}{$23.5[19.3,33.2]$} & \multicolumn{4}{|c|}{$23.5[17.7,34.1]$} & & \\
\hline \multicolumn{12}{|l|}{ Injury intent } \\
\hline Unintentional & 22 & 776 & 426 & 1,372 & 7.9 & 19,998 & 790,532 & 638,015 & 959,658 & 33.0 & 0.0009 \\
\hline Assault & 349 & 8,192 & 7,433 & 8,742 & 83.7 & 56,317 & $1,441,298$ & $1,259,506$ & $1,612,043$ & 60.2 & \\
\hline Suicide & 18 & 580 & 318 & 1,035 & 5.9 & 3,774 & 131,815 & 95,559 & 180,579 & 5.5 & \\
\hline Law enforcement & 11 & 241 & 144 & 401 & 2.5 & 932 & 31,308 & 22,992 & 42,630 & 1.3 & \\
\hline \multicolumn{12}{|l|}{ Firearm type } \\
\hline Handgun & 104 & 2,617 & 2,278 & 2,839 & 83.5 & 24,781 & 701,369 & 587,132 & 816,930 & 48.3 & 0.0001 \\
\hline Rifle & 8 & 287 & 145 & 541 & 9.2 & 3,796 & 145,263 & 110,105 & 189,997 & 10.0 & \\
\hline Shotgun & 5 & 224 & 60 & 732 & 7.1 & 3,256 & 131,436 & 112,865 & 152,666 & 9.0 & \\
\hline BB & 1 & 6 & 1 & 47 & 0.2 & 12,680 & 474,511 & 391,615 & 565,634 & 32.7 & \\
\hline \multicolumn{12}{|l|}{ Hospital size } \\
\hline Small & 7 & 589 & 228 & 1,441 & 5.7 & 6,476 & 507,349 & 363,664 & 690,642 & 19.1 & 0.0061 \\
\hline Medium & 15 & 690 & 257 & 1,727 & 6.7 & 7,430 & 406,425 & 275,406 & 584,308 & 15.3 & \\
\hline
\end{tabular}




\section{Cureus}

\begin{tabular}{|c|c|c|c|c|c|c|c|c|c|c|c|}
\hline Large & 69 & 3,988 & 1,511 & 7,198 & 38.7 & 13,608 & 758,313 & 404,071 & $1,251,025$ & 28.5 & \\
\hline Very large & 313 & 4,937 & 2,426 & 7,553 & 48.0 & 58,525 & 958,628 & 643,589 & $1,326,522$ & 36.1 & \\
\hline Children's & 16 & 92 & 38 & 222 & 0.9 & 4,681 & 27,646 & 18,077 & 42,534 & 1.0 & \\
\hline \multicolumn{12}{|l|}{ Sex } \\
\hline Male & 379 & 9,332 & 8,950 & 9,612 & 90.8 & 78,802 & $2,295,005$ & $2,267,481$ & $2,320,634$ & 86.4 & 0.0033 \\
\hline Female & 39 & 944 & 664 & 1,326 & 9.2 & 11,888 & 362,615 & 336,986 & 390,139 & 13.6 & \\
\hline \multicolumn{12}{|l|}{ Race } \\
\hline White & 80 & 2,830 & 2,241 & 3,481 & 35.1 & 23,843 & 931,455 & 740,477 & $1,133,254$ & 42.6 & 0.059 \\
\hline Black & 146 & 3,053 & 1,803 & 4,540 & 37.9 & 38,409 & 872,340 & 626,255 & $1,144,195$ & 39.9 & \\
\hline Amerindian & 64 & 2,072 & 1,193 & 3,287 & 25.7 & 9,368 & 363,148 & 203,062 & 610,719 & 16.6 & \\
\hline Asian & 6 & 98 & 35 & 271 & 1.2 & 866 & 21,228 & 11,378 & 39,606 & 1.0 & \\
\hline \multicolumn{12}{|l|}{ Incident locale } \\
\hline Home & 68 & 1,969 & 1,510 & 2,461 & 41.6 & 20,327 & 732,937 & 631,178 & 836,432 & 47.1 & 0.011 \\
\hline School/recreation & 14 & 354 & 153 & 775 & 7.5 & 1,986 & 78,502 & 64,005 & 96,242 & 5.0 & \\
\hline Street/highway & 53 & 1,265 & 848 & 1,792 & 26.7 & 18,310 & 434,890 & 316,601 & 576,828 & 27.9 & \\
\hline Other property & 47 & 1,144 & 834 & 1,523 & 24.2 & 10,250 & 304,040 & 252,129 & 363,632 & 19.5 & \\
\hline Farm & 0 & 0 & 0 & 0 & 0.0 & 127 & 6,942 & 4,360 & 11,057 & 0.4 & \\
\hline \multicolumn{12}{|l|}{ Transportation to ED } \\
\hline Emergency medical service & 349 & 8,414 & 7,860 & 8,843 & 84.4 & 49,921 & $1,255,959$ & $1,049,318$ & $1,458,588$ & 52.8 & 0.0001 \\
\hline Air & 27 & 808 & 473 & 1,346 & 8.1 & 1,917 & 58,535 & 34,462 & 98,396 & 2.5 & \\
\hline Private vehicle & 13 & 521 & 272 & 976 & 5.2 & 17,930 & 698,677 & 545,218 & 874,630 & 29.4 & \\
\hline Walk-in & 5 & 144 & 64 & 321 & 1.4 & 8,161 & 283,043 & 203,446 & 388,355 & 11.9 & \\
\hline Police & 5 & 79 & 15 & 418 & 0.8 & 2,977 & 72,219 & 35,413 & 145,217 & 3.0 & \\
\hline Other & 0 & 0 & 0 & 0 & 0.0 & 237 & 8,278 & 4,040 & 16,399 & 0.3 & \\
\hline \multicolumn{12}{|l|}{ Anatomic location of injury } \\
\hline Head/neck & 126 & 3,015 & 3,593 & 306,716 & 29.6 & 25,521 & 793,383 & 740,891 & 847,663 & 30.5 & 0.0004 \\
\hline Upper trunk & 138 & 3,605 & 4,305 & 366,737 & 35.4 & 14,266 & 381,696 & 334,378 & 434,639 & 14.7 & \\
\hline Lower trunk & 148 & 3,427 & 3,894 & 348,629 & 33.7 & 11,123 & 289,804 & 257,815 & 325,003 & 11.1 & \\
\hline Upper extremity & 1 & 82 & 610 & 8,342 & 0.8 & 14,783 & 491,967 & 442,972 & 545,057 & 18.9 & \\
\hline Lower extremity & 4 & 44 & 137 & 4,476 & 0.4 & 23,075 & 647,338 & 615,109 & 680,735 & 24.9 & \\
\hline \multicolumn{12}{|l|}{ Diagnosis } \\
\hline Contusion/abrasion & 0 & 0 & 0 & 0 & 0.0 & 5,069 & 166,538 & 142,625 & 194,201 & 6.3 & $<10-4$ \\
\hline Foreign body & 23 & 576 & 1,222 & 59,305 & 5.6 & 9,135 & 323,836 & 248,409 & 417,875 & 12.3 & \\
\hline Fracture & 163 & 3,752 & 4,747 & 386,306 & 36.4 & 6,523 & 188,859 & 157,624 & 225,779 & 7.2 & \\
\hline Laceration & 34 & 839 & 1,410 & 86,383 & 8.1 & 9,807 & 335,217 & 263,935 & 422,348 & 12.7 & \\
\hline Internal organ injury & 61 & 1,093 & 2,413 & 112,535 & 10.6 & 4,640 & 127,629 & 99,469 & 163,413 & 4.9 & \\
\hline Puncture & 54 & 2,974 & 4,282 & 306,203 & 28.9 & 28,777 & 840,801 & 671,810 & $1,029,951$ & 32.0 & \\
\hline Not stated & 45 & 1,062 & 1,964 & 109,344 & 10.3 & 26,026 & 648,574 & 497,345 & 827,855 & 24.6 & \\
\hline ED Disposition & & & & & & & & & & & \\
\hline
\end{tabular}




\section{Cureus}

\begin{tabular}{|c|c|c|c|c|c|c|c|c|c|c|c|}
\hline Treated and released & 35 & 1,309 & 793 & 2,086 & 12.8 & 51,326 & $1,686,619$ & $1,503,591$ & $1,855,563$ & 64.0 & 0.0006 \\
\hline Admit & 382 & 8,873 & 8,097 & 9,394 & 86.8 & 33,742 & 813,584 & 664,132 & 979,984 & 30.9 & \\
\hline Fatal & 2 & 37 & 9 & 145 & 0.4 & 4,910 & 136,289 & 115,742 & 160,299 & 5.2 & \\
\hline \multicolumn{12}{|l|}{ Marital Status } \\
\hline Never married & 150 & 4,017 & 3,488 & 4,442 & 74.0 & 33,207 & 927,325 & 810,059 & $1,029,794$ & 68.0 & 0.20 \\
\hline Married & 26 & 910 & 585 & 1,364 & 16.8 & 7,619 & 308,953 & 244,696 & 384,366 & 22.7 & \\
\hline Divorced/separated & 7 & 173 & 96 & 308 & 3.2 & 1,564 & 60,117 & 44,329 & 81,156 & 4.4 & \\
\hline Other & 6 & 327 & 87 & 1,093 & 6.0 & 1,239 & 67,661 & 24,961 & 173,906 & 5.0 & \\
\hline \multicolumn{12}{|l|}{ Argument } \\
\hline Yes & 30 & 515 & 193 & 1,126 & 19.6 & 5,440 & 165,558 & 136,973 & 198,871 & 15.0 & 0.54 \\
\hline No & 69 & 2,115 & 1,504 & 2,437 & 80.4 & 25,064 & 941,741 & 908,428 & 970,326 & 85.0 & \\
\hline \multicolumn{12}{|l|}{ Crime } \\
\hline Yes & 61 & 1,319 & 908 & 1,775 & 40.6 & 15,238 & 352,085 & 230,102 & 507,398 & 27.6 & 0.046 \\
\hline No & 56 & 1,926 & 1,470 & 2,337 & 59.4 & 13,695 & 923,426 & 768,113 & $1,045,409$ & 72.4 & \\
\hline \multicolumn{12}{|l|}{ Illicit drug involvement } \\
\hline Yes & 35 & 958 & 709 & 1,242 & 34.7 & 2,457 & 83,146 & 46,498 & 144,475 & 8.0 & 0.0052 \\
\hline No & 60 & 1,804 & 1,520 & 2,053 & 65.3 & 25,781 & 954,749 & 893,420 & 991,397 & 92.0 & \\
\hline \multicolumn{12}{|l|}{ Fight } \\
\hline Yes & 36 & 872 & 555 & 1,291 & 25.1 & 7,768 & 229,151 & 189,007 & 275,510 & 19.0 & 0.20 \\
\hline No & 83 & 2,609 & 2,190 & 2,926 & 74.9 & 25,879 & 973,951 & 927,592 & $1,014,095$ & 81.0 & \\
\hline \multicolumn{12}{|l|}{ Alcohol involvement } \\
\hline Yes & 29 & 818 & 521 & 1,261 & 7.9 & 4,081 & 143,694 & 87,538 & 236,836 & 5.4 & 0.056 \\
\hline No & 391 & 9,478 & 9,035 & 9,775 & 92.1 & 86,636 & $2,514,519$ & $2,448,377$ & $2,597,675$ & 93.6 & \\
\hline \multicolumn{12}{|l|}{ Sexual assault } \\
\hline Yes & 0 & 0 & 0 & 0 & 0.0 & 505 & 13,067 & $2,640,018$ & $2,649,057$ & 0.5 & 0.0012 \\
\hline No & 420 & 10,296 & 7,205 & 14,944 & 100.0 & 90,214 & $2,645,278$ & 0 & 0 & 99.5 & \\
\hline \multicolumn{12}{|l|}{ Who caused } \\
\hline Unknown & 260 & 5,773 & 4,879 & 6,578 & 56.3 & 44,887 & $1,103,518$ & 971,206 & $1,227,885$ & 41.7 & 0.028 \\
\hline Stranger & 54 & 1,400 & 1,083 & 1,776 & 13.7 & 13,311 & 369,553 & 314,307 & 428,240 & 14.0 & \\
\hline Self & 30 & 1,017 & 627 & 1,596 & 9.9 & 17,077 & 683,502 & 558,034 & 817,357 & 25.9 & \\
\hline Friend/acquaintance & 19 & 520 & 281 & 935 & 5.1 & 5,425 & 180,846 & 152,792 & 211,476 & 6.8 & \\
\hline Spouse/ex & 2 & 59 & 12 & 281 & 0.6 & 522 & 19,137 & 15,332 & 23,527 & 0.7 & \\
\hline Other relative & 5 & 135 & 60 & 294 & 1.3 & 2,065 & 76,853 & 61,593 & 94,636 & 2.9 & \\
\hline Other/not seen & 50 & 1,392 & 1,008 & 1,875 & 13.6 & 7,433 & 224,952 & 184,778 & 270,161 & 8.5 & \\
\hline
\end{tabular}

TABLE 1: Demographics of those with and without a spine injury and firearm use

$\mathrm{n}=$ actual number of ED visits, $\mathrm{N}=$ estimated number of $\mathrm{ED}$ visits, $\mathrm{L} 95 \%=$ lower $95 \% \mathrm{CI}, \mathrm{U} 95 \%=\mathrm{upper} 95 \% \mathrm{CI}$

ED: emergency department 


\section{Cureus}

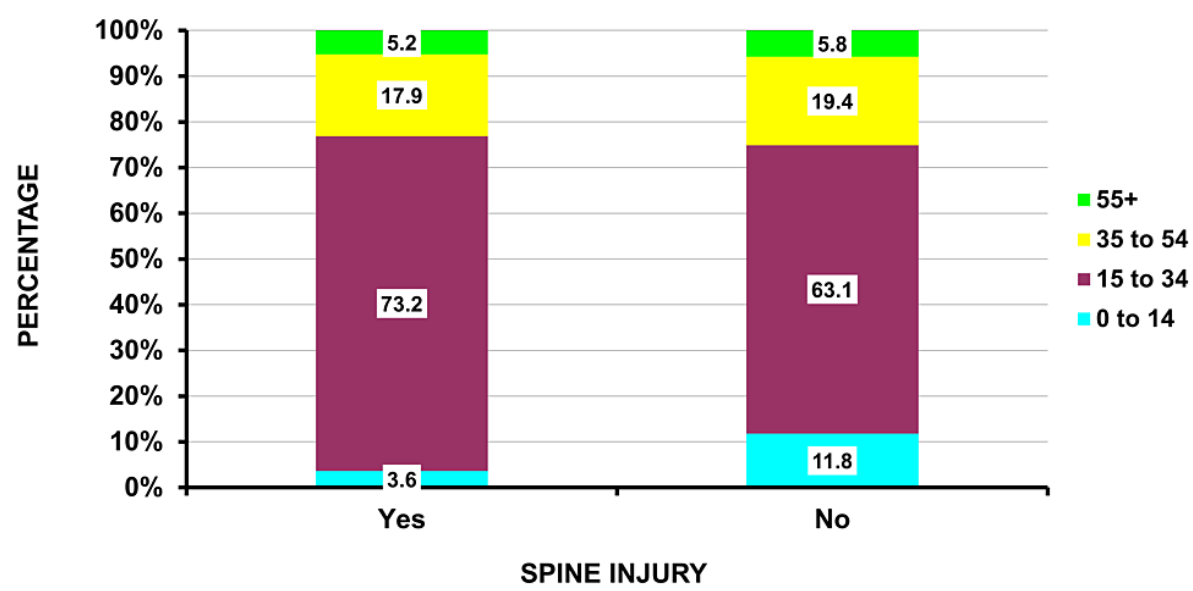

FIGURE 1: Age groupings for those with and without spine injury due to firearms $(p=0.0001)$

The actual percentages are shown in each cell.

\section{Patients with spinal injury}

The anatomic location within the spine was identified in 10,197 (99.0\%) of the injuries. The spinal level was $32 \%$ thoracic $(3,325), 32 \%$ lumbar $(3,213), 30 \%$ cervical $(3,050)$, and $6 \%$ sacrococcygeal $(609)$. The majority $(91.8 \%)(9,438-\{8,863-9,793\})$ of the patients sustained a fracture. There were no differences between patients with and without a fracture by any of the variables in Table 1 or by spine level. We also compared those with and without a neurologic injury. Patients without a fracture were more likely to have sustained a neurologic injury ( 97.9 vs. $79.1 \%$ - $\mathrm{p}=0.0037$ ), and there was a significant difference in neurologic injury by spine level (Figure 2). Patients with injuries to the thoracic area had the highest percentage of neurologic involvement (50.4\%), followed by the lumbar spine (28.6\%) and the cervical spine (24.7\%).

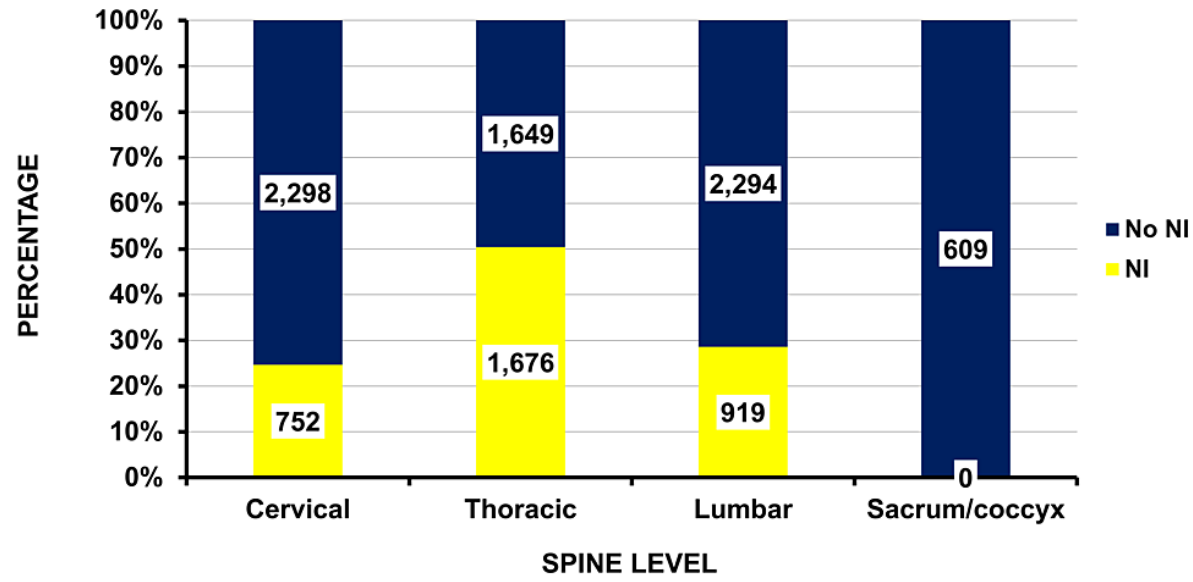

FIGURE 2: Differences by spine level with or without neurologic injury (NI) $(p=0.0003$ includes sacrum/coccyx, and $p=0.01$ excludes the sacrum/coccyx). The estimated numbers are given in each cell.

\section{Non-powder firearm gunshot wound injuries}

Although the majority (97.0\%) of patients with spinal injuries associated with firearms involved a powder firearm gunshot wound, $2.9 \%$ involved a powder firearm without a gunshot wound. There was one case involving an air-powered firearm, indicating that air-powered weapons can also result in injury. To further explore this issue, the narrative comments of the actual (not estimated) 420 spine injury cases were reviewed to obtain an idea of the types of powder firearm non-gunshot wound injuries. There were 16 actual cases involving powder firearms without a gunshot wound. Four of these were due to falls from hunting stands 


\section{Cureus}

resulting in spine fractures. The others were due to various assaults resulting in various injuries such as "the patient was assaulted with the handle of a 38-caliber handgun resulting in a closed head injury and C1 fracture." Another example is "the patient was assaulted by multiple people and pistol-whipped, resulting in L2, 3, 4 fractures, and hemopneumothorax with rib fractures.” The single air-powered firearm wound occurred when a 12-year-old child was shot in the posterior thoracic area by his brother with a pellet gun, with the pellet lodged in the T11 neural foramen.

\section{Changes over time}

Joinpoint regression demonstrated an annual percentage decrease for both those patients with and without spine involvement in the 1990s, followed by increases through 2015. The average percentage increase for patients with a spine injury was 10.3\% per year from 1997 onwards $(\mathrm{p}<10-6)$ (Figure 3), significantly greater than the $1.5 \%$ for those without spinal involvement $(\mathrm{p}=0.0001)$ from 1999 onward (Figure 4$)$.

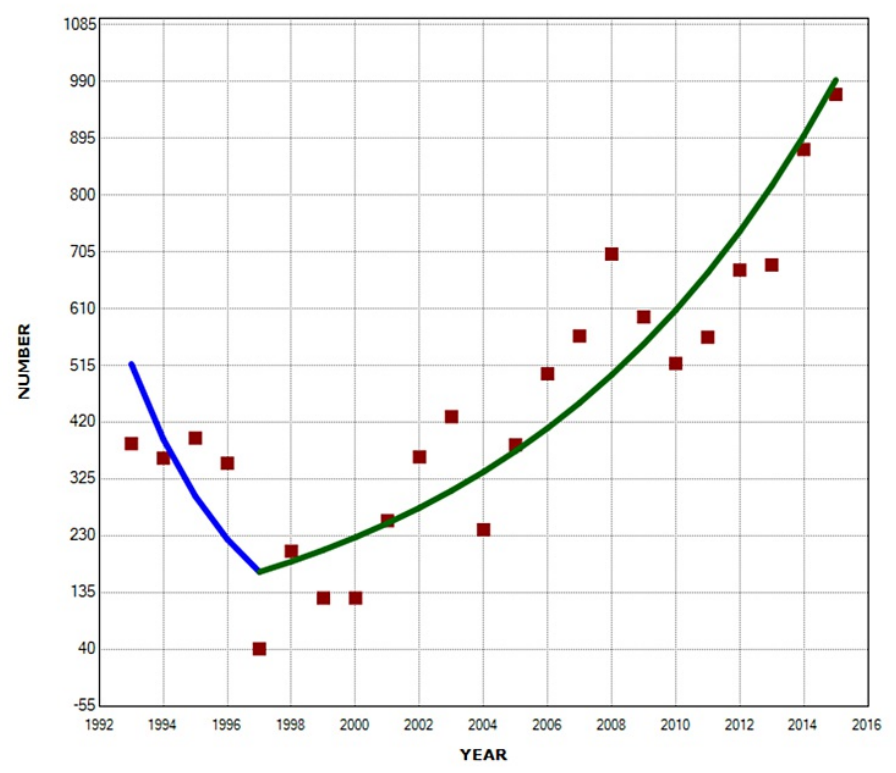

FIGURE 3: Joinpoint regression analyses or those with a spine injury

There was an annual decrease of $24.3 \%$ from 1993 through $1997(p=0.016)$, and then an annual increase of $10.3 \%$ from 1997 through 2015 ( $p<10-6)$. 


\section{Cureus}

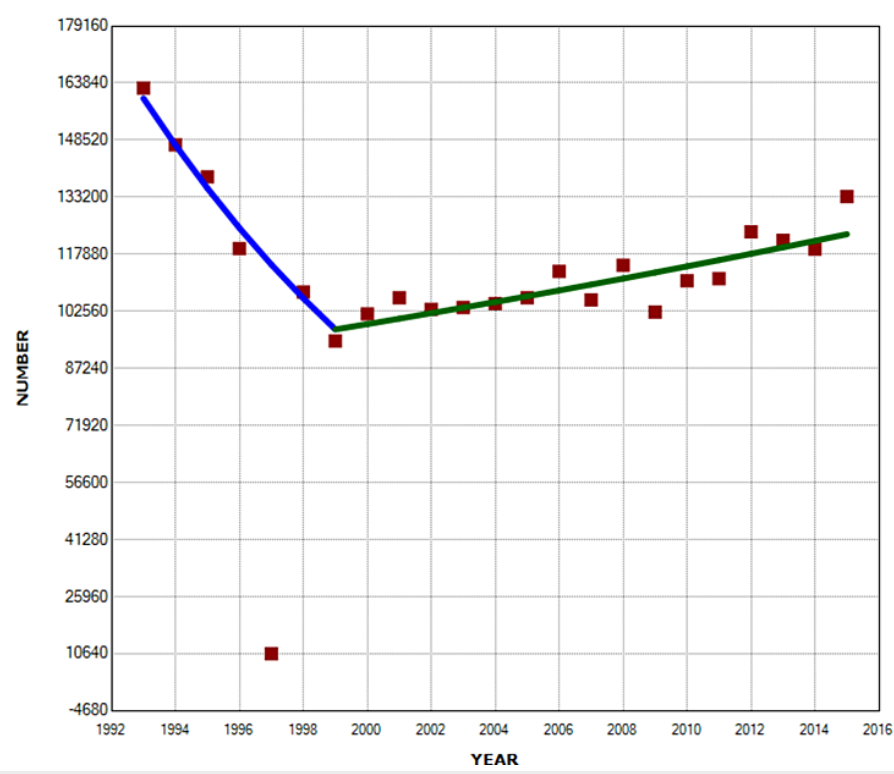

\section{FIGURE 4: Joinpoint analyses for those without a spine injury}

There was an average annual decrease of $7.9 \%$ from 1993 to $1999(p=0.0002)$, and then an average annual increase of $1.5 \%$ from 1999 through $2015(p=0.0001)$.

\section{Discussion}

There are few studies that allow us to compare the results of our present study. A compilation of the literature regarding civilian firearm injuries to the spine finds similar findings to those in this study (Table 2). Excluding those studies of only children, the average age was similar: 28 years in this study and 25 to 27 in the others [9-12]. The vast majority of the patients were male: $91 \%$ in this study and $80 \%$ to $94 \%$ in the literature [9-13]. The anatomic location of the injury was also similar (Figure 5). 


\section{Cureus}

\begin{tabular}{|c|c|c|c|c|c|c|c|c|c|}
\hline & $\begin{array}{l}\text { Present } \\
\text { Study }\end{array}$ & $\begin{array}{l}\text { Turgut } \\
{[9] \$}\end{array}$ & $\begin{array}{l}\text { Rukovansjki } \\
\text { [14] }\end{array}$ & $\begin{array}{l}\text { Carillo } \\
{[13]^{\wedge}}\end{array}$ & $\begin{array}{l}\text { de Amoreira Gepp } \\
{[15]^{\wedge} \$}\end{array}$ & Fife [16]\$ & Rhee [10] & $\begin{array}{l}\text { Trahan } \\
\text { [11] }\end{array}$ & $\begin{array}{l}\text { Waters } \\
{[12] \$}\end{array}$ \\
\hline$n^{\star}$ & 10,296 & 17 & 20 & 19 & 11 & 73 & 168 & 147 & 135 \\
\hline GSW alone & $\mathrm{N}$ & Y & Y & Y & Y & $\mathrm{N}$ & $\mathrm{N}$ & Y & Y \\
\hline $\mathrm{SCl}$ alone & $\mathrm{N}$ & Y & Y & Y & Y & Y & $\mathrm{N}$ & $\mathrm{N}$ & Y \\
\hline $\begin{array}{l}\text { Geographic } \\
\text { location }\end{array}$ & All USA & Turkey & Croatia & Miami & Brazil & California & $\begin{array}{l}\text { LA, Wash } \\
\text { DC }\end{array}$ & $\begin{array}{l}\text { New } \\
\text { Orleans }\end{array}$ & California \\
\hline Years studied & 1993-2015 & $\begin{array}{l}1968- \\
1990\end{array}$ & 1991-1993 & $\begin{array}{l}1992- \\
1995\end{array}$ & 1996-2009 & $\begin{array}{l}1970- \\
1971\end{array}$ & $1993-2000$ & $\begin{array}{l}2007- \\
2011\end{array}$ & NA \\
\hline \multicolumn{10}{|l|}{ Age (yrs) } \\
\hline Average & 28 & 25 & & 17 & & - & 26 & 27 & 25 \\
\hline Range & $<1$ to 112 & $16-40$ & 12 to 57 & 14-19 & $0-10$ & - & & $14-66$ & $17-59$ \\
\hline$\%$ Male & 91 & 82 & 80 & 95 & - & - & 92 & 92 & 94 \\
\hline \multicolumn{10}{|l|}{ Injury intent } \\
\hline Unintentional & 8 & 6 & & - & - & - & - & - & - \\
\hline Assault & 84 & 82 & 100 & - & - & - & - & - & - \\
\hline Self & 6 & 12 & & - & - & - & - & - & - \\
\hline \multicolumn{10}{|l|}{ Spine level (\%) } \\
\hline Cervical & 30 & 47 & 40 & 16 & 18 & 37 & 100 & 27 & 19 \\
\hline Thoracic & 32 & 18 & 40 & 21 & 73 & 48 & 0 & 36 & 52 \\
\hline Lumbosacral & 38 & 35 & 35 & 63 & 9 & 15 & 0 & 36 & 29 \\
\hline \multicolumn{10}{|l|}{ Race (\%) } \\
\hline White & 35.1 & - & - & - & - & - & - & 9.0 & 4.4 \\
\hline Black & 37.9 & - & - & - & - & - & - & 84.0 & 46.7 \\
\hline Amerindian & 25.7 & - & - & - & - & - & - & & 45.2 \\
\hline Asian & 1.2 & - & - & - & - & - & - & & \\
\hline $\begin{array}{l}\text { Drug } \\
\text { involvement }\end{array}$ & 35 & - & - & 37 & - & - & - & 39 & \\
\hline $\begin{array}{l}\text { Alcohol } \\
\text { involvement }\end{array}$ & 0 & - & - & 26 & - & - & - & 16 & \\
\hline
\end{tabular}

\section{TABLE 2: Literature comparison of spinal injuries due to firearms}

$\mathrm{N}=$ no, $\mathrm{Y}=$ yes, GSW = gunshot injury, $\mathrm{SCl}=$ spinal cord injury

* the $\mathrm{n}$ is for only those with GSW $\mathrm{s}$ in each study

$\wedge$ only children

\$ only those with spinal cord injuries; the others include both those with and without spinal cord injuries 


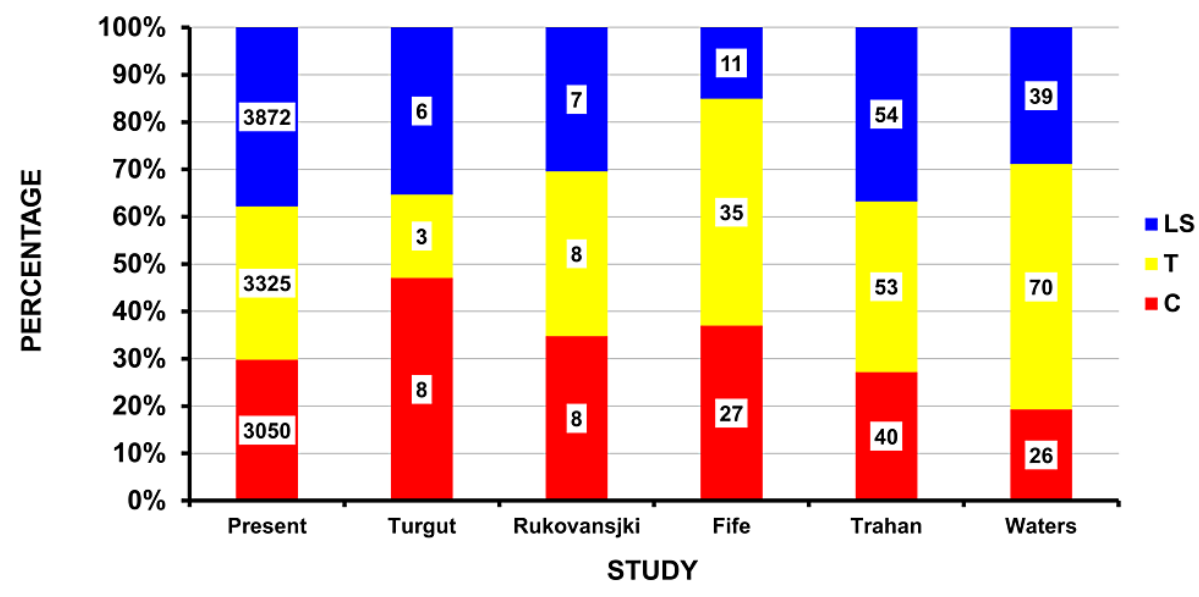

FIGURE 5: Location of spine injury due to firearms: present study and those in the literature

The actual number of cases is shown in each cell.

The vast majority (86.7\%) of the patients with a spine injury were seen in large or very large hospitals (Table 1). This pattern likely indicates firearm injury due to urban violence [17-21], supported by the fact that $83.7 \%$ of the patients with a spine injury were injured during an assault. Although the number of spinal firearm injuries initially decreased in the 1990s, there was an annual 10.3\% increase from 1997 through 2015. This likely reflects the epidemic of increasing firearm violence [4,22-23].

Non-powder weapons can result in serious injury [24-26], especially in children and adolescents. These injuries include blindness and paralysis [25], subarachnoid hemorrhage; lung, liver, and kidney lacerations; pulmonary artery injury; and tracheal injury [24], with 30\% requiring an operative procedure [26]. One case in this study involved a 12-year-old child having a pellet gun missile becoming lodged in the T11 neural foramen, which is a significant injury and required hospital admission.

A neurologic injury was most frequent when the firearm injury involved the thoracic spine. This is likely due to the fact that cervical spine injuries, especially those involving the upper cervical spine (e.g. C1-4) can easily result in immediate/rapid death. It is possible that such a patient was never taken to an ED but was rather pronounced dead at the scene and transferred to the morgue. The proportion of spinal injuries between the cervical, thoracic, and lumbar areas was very similar. This is surprising because the available anatomic height differs among the different spinal regions, with the cervical spine having a smaller height than the thoracic or lumbar spine. The reason that the cervical spine had relatively equal numbers is unknown. One hypothesis is that perhaps the perpetrator was firing towards the head, but the bullet hit the cervical spine instead.

The demographics of firearm injuries point to potential prevention strategies for such injuries. In this study, 90.8\% involved males, $83.7 \%$ an assault, $83.5 \%$ a handgun, $73.2 \%$ were 15 to 34 years, with many also involving a crime (40.6\%) or drug activity (34.7\%). Focusing interventions on these high-risk demographic groups is one prevention approach. Handgun control has certain efficacy [27] but is presently a very politically charged issue in the United States; how gun control laws may change in the future is unknown. Also, illegal handgun use is difficult to control [28]. In Philadelphia, reclaiming blighted vacant urban land significantly reduced shootings that resulted in serious injury or death between the years 2013 to 2015 [19]. Events involving a crime or drug activity are likely codependent; reducing illicit drug activity would hopefully result in less criminal activity as well.

The limitations of this study must be acknowledged. First is the accuracy of the NEISS data. However, previous studies [29-30], including those involving firearms, have demonstrated an over 90\% accuracy of NEISS data. Next, the NEISS only identifies individuals who sought care in an ED. It does not include those who might have been treated in urgent care centers, physician offices, other venues, or those who did not seek any medical care. However, any person sustaining a spinal injury due to a firearm would likely present to an ED. Thus, the data presented in this study are likely very accurate. The NEISS does not allow for analyses by the socioeconomic status of the injured patient, nor detailed geographic regions (i.e. exactly which city and where in a particular city) but does allow for analyses by hospital size, which is a proxy of rural versus urban locations. Finally, the NEISS does not give details regarding treatment and outcomes except for disposition from the ED (release, admit, death). Acknowledging these limitations, the data led to the many interesting results noted above. 


\section{Conclusions}

The vast majority (98.2\%) of spine injuries from firearms were due to powder firearm gunshot wounds. The average age was 28 years with very few $<14$ years of age. The cervical spine was involved in $30 \%$, thoracic in $32 \%$, lumbar in $32 \%$, and sacrum in $6 \%$. A fracture occurred in $91.8 \%$ and neurologic injury in $33 \%$. Injuries to the thoracic spine had the highest percentage of neurologic involvement (50.4\%). This very large US-wide study of spinal injuries associated with firearms covering all ages can be used as baseline data for future firearm studies. The need for firearm injury research has been recently noted. A reduction in the incidence of such injuries can be guided by our findings, although it may be difficult. The relentless rise of $10.3 \%$ per year in firearm spine injuries is certainly a cause for concern.

\section{Additional Information \\ Disclosures}

Human subjects: Consent was obtained or waived by all participants in this study. Indiana University issued approval NA. This study uses publicly de-identified data collected by the National Electronic Injury Surveillance System. This study was considered to be exempt by our local Institutional Review Board. Animal subjects: All authors have confirmed that this study did not involve animal subjects or tissue. Conflicts of interest: In compliance with the ICMJE uniform disclosure form, all authors declare the following: Payment/services info: All authors have declared that no financial support was received from any organization for the submitted work. Financial relationships: All authors have declared that they have no financial relationships at present or within the previous three years with any organizations that might have an interest in the submitted work. Other relationships: All authors have declared that there are no other relationships or activities that could appear to have influenced the submitted work.

\section{References}

1. Choron RL, Spitzerb S, Sakran JV: Firearm violence in America. Is there a solution? . Adv Surg. 2019, 53:195208. 10.1016/j.yasu.2019.04.019

2. Bulger EM, Kuhls DA, Campbell BT, et al.: Proceedings from the medical summit on firearm injury prevention: a public health approach to reduce death and disability in the US. J Am Coll Surg. 2019, 229:415430. 10.1016/j.jamcollsurg.2019.05.018

3. Tasigiorgos S, Economopoulos KP, Winfield RD, Sakran JV: Firearm injury in the United States: an overview of an evolving public health problem. J Am Coll Surg. 2015, 221:1005-1014.

10.1016/j.jamcollsurg.2015.08.430

4. Ranney ML, Herges C, Metcalfe L, Schuur JD, Hain P, Rowhani-Rahbar A: Increases in actual health care costs and claims after firearm injury. Ann Int Med. 2020, 173:949-955. 10.7326/M20-0323

5. Richmond TS, Lemaire J: Years of life lost because of gunshot injury to the brain and spinal cord . Am J Phys Med Rehabil. 2008, 87:609-615. 10.1097/PHM.0b013e31817fb496

6. Chien L-C, Gakh M, Coughenour C, Lin R-T: Temporal trend of research related to gun violence from 1981 to 2018 in the United States: a bibliometric analysis. Injury Epidemiology. 2020, 7:9. 10.1187/s40621-0200235-6

7. US Department of Health and Human Services, Centers for Disease Control and Prevention, National Center for Injury Prevention and Control, United States Consumer Product Safety Commission. Firearm Injury Surveillance Study, 1993-2015. Inter-University Consortium for Political and Social Research. (2021). http://www.icpsr.umich.edu.

8. Eveleth PB, Tanner JM: Worldwide Variation in Human Growth. Cambridge University Press, Cambridge, England; 1990.

9. Turgut M, Özcan ÖE, Gürcay Ö, Sağlam S: Civilian penetrating spinal firearm injuries of the spine. Results of surgical treatment with special attention to factors determining prognosis. Arch Orthop Trauma Surg. 1994, 113:290-293. 10.1007/BF00443820

10. Rhee P, Kuncir EJ, Johnson L, et al.: Cervical spine injury is highly dependent on the mechanism of injury following blunt and penetrating assault. J Trauma. 2006, 61:1166-1170. 10.1097/01.ta.0000188163.52226.97

11. Trahan J, Serban D, Tender GC: Gunshot wounds to the spine in post-Katrina New Orleans . Injury. 2013, 44:1601-1606. 10.1016/j.injury.2013.06.021

12. Waters RL, Adkins RH, Yakuram J, Sie I: Profiles of spinal cord injury and recovery after gunshot injury . Clin Orthop Rel Res. 1991, 267:14-21.

13. Carrillo EH, Gonzalez JK, Carrillo LE, Chacon PM, Namias N, Kirton OC, Byers PM: Spinal cord injuries in adolescents after gunshot wounds: an increasing phenomenon in urban North America. Injury. 1998, 29:503-507. 10.1016/s0020-1383(98)00110-7

14. Rukovansjki M: Spinal cord injuries caused by missile weapons in the Croatian war . J Trauma. 1996, 40:189192. 10.1097/00005373-199603001-00042

15. de Amoreira Gepp R, Nadal LG: Spinal cord trauma in children under 10 years of age: clinical characteristics and prevention. Child Nerv Sys. 2012, 28:1919-1924. 10.1007/s00381-012-1846-1

16. Fife D, Kraus J: Anatomic location of spinal cord injury. Relationship to the cause of injury . Spine. 1986, 11:2-5. 10.1097/00007632-198601000-00002

17. Roberto E, Braga AA, Papachristos AV: Closer to guns: the role of street gangs in facilitating access to illegal firearms. J Urban Health. 2018, 2018:372-38210. 10.1007/s11524-018-0259-1

18. Beard JH, Morrison CN, Jacoby SF, Dong B, Smith R, Sims CA, Wiebe DJ: Quantifying disparities in urban firearm violence by race and place in Philadelphia, Pennsylvania: a cartographic study. Am J Pub Health. 2017, 107:371-373. 10.2105/AJPH.2016.303620

19. Moyer R, MacDonald JM, Ridgeway G, Branas CC: Effect of remediating blighted vacant land on shootings: a 


\section{Cureus}

citywide cluster randomized trial. Am J Pub Health. 2019, 109:140-144. 10.2105/AJPH.2018.304752

20. Beard JH, Resnick S, Maher Z, et al.: Clustered arrivals of firearm-injured patients in an urban trauma system: a silent epidemic. J Am Coll Surg. 2019, 229:236-243. 10.1016/j.jamcollsurg.2019.03.020

21. Tobon M, Ledgerwood AM, Lucas CE: The urban injury severity score (UISS) better predicts mortality following penetrating gunshot wounds (GSW). Am J Surg. 2019, 217:537-576. 10.1016/j.amjsurg.2018.09.013

22. McLean RM, Harris P, Cullen J, Maier RV, Yasuda KE, Schwartz BJ, Benjamin GC: Firearm-related injury and death in the United States: a call to action from the nation's leading physician and public health professional organizations. Ann Int Med. 2019, 171:573-577. 10.7326/M19-2441

23. Kochanek KD, Murphy SL, Xu J, Arias E: Deaths: final data for 2017. Natl Vital Stat Rep. 2019, 68:1-77.

24. Hyak JM, Todd H, Rubalcava D, Vogel AM, Fallon S, Naik-Mathuria B : Barely benign: the dangers of BB and other nonpowder guns. J Ped Surg. 2020, 55:1604-1609. 10.1016/j.jpedsurg.2020.02.010

25. McLoughlin RJ, Stetson A, Green J, Hirsh MP, Cleary MA, Aidlen JT: Toy guns, real danger: an update on pediatric injury patterns related to nonpowder weapons. J Ped Surg. 2020, 55:146-152. 10.1016/j.jpedsurg.2019.09.068

26. Apelt N, Greenwell C, Tweed J, et al.: Air guns: a contemporary review of injuries at six pediatric level I trauma centers. J Surg Res. 2020, 248:1-6. 10.1016/j.jss.2019.11.002

27. Luca M, Malhotra D, Poliquin C: Handgun waiting periods reduce gun deaths . Proc Nat Acad Sci. 2017, 114:12162-12165. 10.1073/pnas.1619896114

28. Chesnut KY, Barragan M, Gravel J, Pifer NA, Reiter K, Sherman N, Tita GE: Not an 'iron pipeline', but many capillaries: regulating passive transactions in Los Angeles’ secondary, illegal gun market. Inj Prev. 2017, 23:226-231. 10.1136/injuryprev-2016-042088

29. Annest JL, Mercy JA, Gibson DR, Ryan GW: National estimates of nonfatal firearm-related injuries. Beyond the tip of the iceberg. JAMA. 1995, 273:1749-1754. 10.1001/jama.1995.03520460031030

30. Hopkins RS: Consumer product-related injuries in Athens, Ohio, 1980-1985: assessment of emergency room-based surveillance. Am J Prev Med. 1989, 5:104-112. 10.1016/S0749-3797(18)31112-7 